\title{
Multidisciplinary Design Optimization of Distribution Cam Mechanism of Diesel Engine
}

\author{
Li Lei ${ }^{1,2, *}$ and Zhang Jianrun ${ }^{2}$ \\ ${ }^{1}$ College of Mechanical Engineering, Southeast University, 211189 Nanjing, China \\ ${ }^{2}$ College of Mechanical Engineering, Jiangsu University of Science and Technology, 212000 Zhenjiang, China
}

Received: 27 Jan. 2013, Revised: 28 May. 2013, Accepted: 29 May. 2013

Published online: 1 Sep. 2013

\begin{abstract}
Multidisciplinary Design Optimization (MDO) has emerged as a new technology dealing with the design of complex systems. MDO is employed in designing of diesel engine structure for improving the system performance in this paper. According to the analysis of the Distribution Cam Mechanism (DCM), ventilation, vibration and mechanical performance are selected as indicators. Basing kinematic analysis by ADAMS, the fullness coefficient, contact stress and the maximum acceleration performances are obtained. Under the environment of MDO, multi-objective genetic algorithm is used to overcome the difficulty of convergence in optimization of the DCM and obtain the best Pareto solution set. In addition, approximate model is proposed to improve the design process and proved to be efficiency. Finally, the results of multi-objective optimization are compared to single-objective optimization, which demonstrates that fullness coefficient and contact stress of the diesel engine DCM is improved greatly without reduce the ventilation performance. The effective and efficient collaboration mechanism for coordinating coupled disciplines in the process of diesel engine design and optimization is developed in this research, which owns a high theory and application value.
\end{abstract}

Keywords: Multidisciplinary, Cam Mechanism, Diesel Engine

\section{Introduction}

Multidisciplinary Design Optimization (MDO) can take advantage of the interactions between disciplines as well as to improve the product development time and has emerged as a new technology dealing with the design of complex systems[1]. MDO techniques have been in development over the last decade and were applied primarily to design of aeronautics, astronautics and automobile [2-6]. However, the application of MDO method to the diesel engine design has not been achieved so far.

Distribution cam mechanism (DCM) determines the power, economy and reliability performance of the diesel engine, and has a direct impact on the vibration and noise performance of the whole diesel engine system. In general, cam shape line was selected as the optimization goal to meet the intake and exhaust requirements of the diesel engine: Hao Zhiyong [7] invented an optimizing template of high-ordered polynomial cam shape line and conducted an optimal calculation to a high duty diesel engine with four valves and double over- head cam; Long
Lianchun [8] established an optimization design model based on a three-freedom analysis model taking the time-area of cam profile as objective function, subjects to the demands of dynamic and strength characteristics; Li Zhi [9] did the dynamic simulation of distribution cam mechanism in internal combustion engine based on ant colony algorithm. Because the optimization process involves coupled multiple disciplines, traditional methods for single target are no longer meet the requirement of improving the overall performance of diesel engine. Therefore, MDO for multi objective is imported into diesel engine design in this paper. As the application of MDO method is restricted by the high computational cost of running the simulation codes in the optimization procedure, numerous approaches have been proposed for balancing computational efficiency and analysis accuracy: M.A. Schònning [10] utilized a dependency tracking language to reduce computational time during multidisciplinary design optimization; the common method is to apply the approximation models such as response surface models (RSM) [11] in conjunction with design of experiment (DOE)[12], Taylor series

\footnotetext{
*Corresponding author e-mail: lilei0064@ sina.com
} 
approximations, Variable complexity models, and artificial neural networks[10].

In this article, MDO method for multiobjective (ventilation, vibration and mechanical performance) is imported into the design of diesel engine DCM. Fullness coefficient, contact stress and the maximum acceleration performances are obtained basing kinematic analysis; Multi-objective genetic algorithm is used to overcome the difficulty of convergence in optimization of the DCM and obtain the best Pareto solution set under the environment of MDO; Approximate model is employed in order to improve the efficiency. The results indicate that the performance of the diesel engine gets better and the optimization process owns great efficiency.

\section{Multidisciplinary Analysis of Distribution Cam Mechanism in Diesel Engine}

Aim to obtain the best power and economy performance of the diesel engine, larger valve opening area and best valve time at different speed are required. At the same time, the problem of wear, vibration and noise is caused by inertial force, so that dynamic analysis should be applied at the design stage. In this paper, virtual prototyping of the diesel engine DCM is founded in ADAMS, and the kinematics and dynamics performance are obtained considering the flexibility of different structure.

\subsection{Modeling for Simulation Analysis}

The three-dimensional (3-D) model of a type of diesel engine DCM is shown in Figure 1 whose rated crankshaft speed is $2165 \mathrm{rpm}$. In order to improve the accuracy of analysis, the tappet and rocker are considered to be flexible. And the other constraints of the system are listed in Table 1.

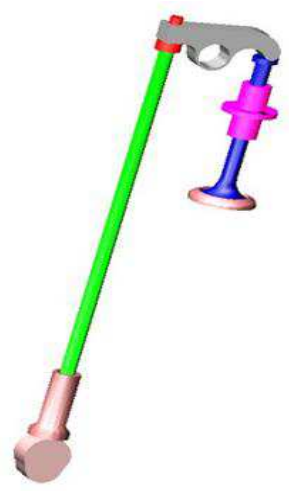

Figure 1: Three-dimensional model of the distribution cam mechanism

\begin{tabular}{|c|c|}
\hline Objects of Constraint & Constraint Type \\
\hline cam and ground & Revolute Joint \\
\hline tappet and cam & Contact \\
\hline tappet and ground & Translational Joint \\
\hline tappet and push rod & Spherical Joint \\
\hline push rod and nut & Spherical Joint \\
\hline nut and push rod & Fixed Joint \\
\hline rocker and ground & Revolute Join \\
\hline rocker and valve & Contact \\
\hline air lock block and intake valve & Fixed Joint \\
\hline valve guides and ground & Fixed Joint \\
\hline valve spring retainer and ground & Fixed Joint \\
\hline intake valves and valve guides & Translational Joint \\
\hline intake valve and intake valve seat & Contact \\
\hline
\end{tabular}

\subsection{Simulation and Results}

Basing on dynamic analyses, the lift, velocity and acceleration of the valve in diesel engine can be obtained. The purpose is to get the contact force between cam and tappet. The detail of simulation analysis is explained below:

2.2.1 Analysis intake and exhaust performance and kinematic analysis of the distribution cam mechanism

Figure 2 shows the intake valve lift curve, and the largest point is $11.082 \mathrm{~mm}$. It can be noticed that the curve is smooth, which means that the valve movement is continuous.

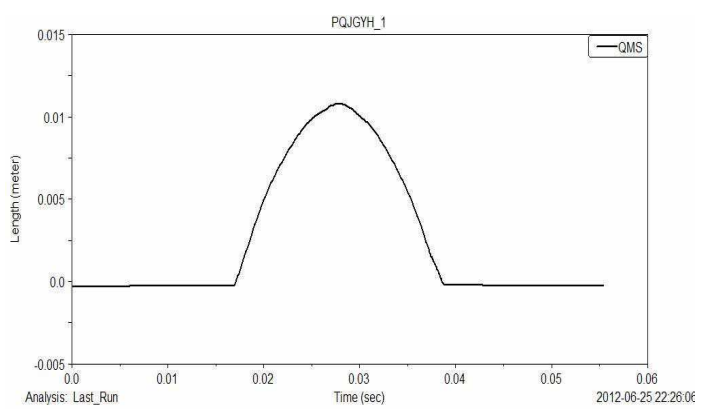

Figure 2: Intake valve lift curve

Intake performance is one of the most important indicators, which can be measured by the fullness coefficient. The formula of fullness coefficient is shown in equation (1):

$$
\xi=\int_{\alpha}^{\beta} \frac{y(a)}{y_{\max }(\alpha-\beta)} d a
$$

Where: $\alpha$ is the camshaft corner at the time of valve opening, $\beta$ is the camshaft corner at the time of valve closing, $y_{\max }$ is the largest valve lift.

Integrating the curve shown in Figure 2 from the opening point to the closing point of the valve basing formula (1), the fullness coefficient of intake valve is 
calculated 0.625. And the fullness coefficient will be selected as the optimization goal in the design of the cam curve linear. Generally, large fullness coefficient is benefit to improve the intake performance, but affect the stability of the distribution cam mechanism. Therefore, the dynamic performance must be considered.

The valve velocity curve and valve acceleration curve are shown in Figure 3 and Figure 4. It can be noticed that the maximum intake valve speed is $1.7688 \mathrm{~m} / \mathrm{s}$, the minimum speed is $-1.799 \mathrm{~m} / \mathrm{s}$, the maximum valve acceleration is $11528.3 \mathrm{~m} / \mathrm{s}^{2}$ and the minimum acceleration is $-667.79 \mathrm{~m} / \mathrm{s}^{2}$.

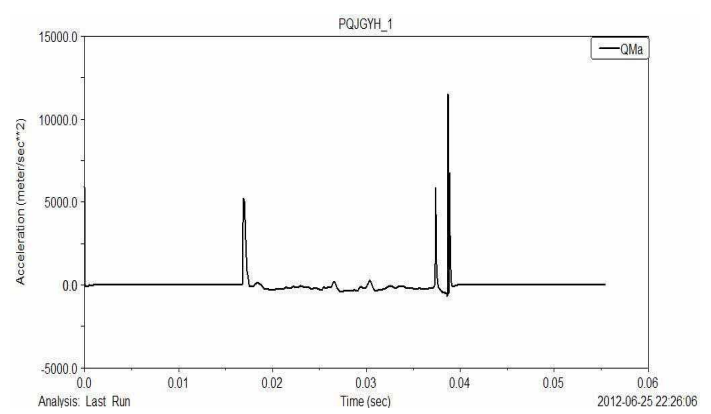

Figure 3: Valve velocity curve

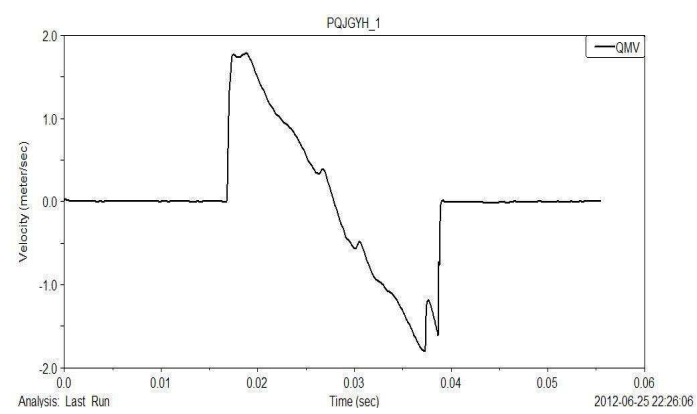

Figure 4: Valve acceleration curve

\subsubsection{Dynamic analysis of the cam and tappet}

Because large contact stress causes wear of the cam, the contact force needs to be analyzed. As shown in Figure 5: force conditions of the cam and tappet is complex, and the largest force is $6073.837 \mathrm{~N}$ at the opening point of the intake valve.

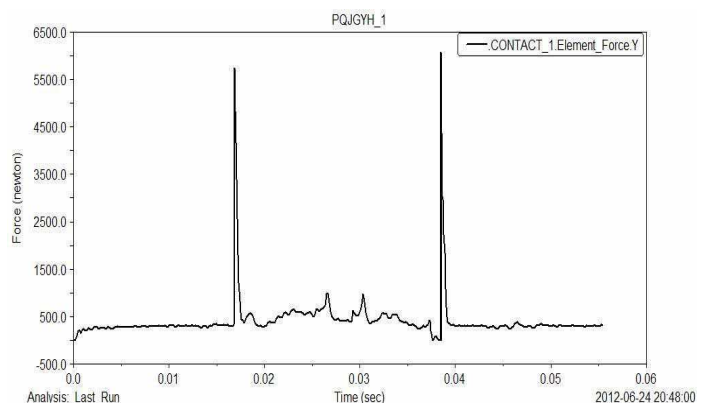

Figure 5: Contact forces of the cam and tappet
Contact of cam and tappet is an important friction pair in diesel engine, which can lead to wear, fracture and other problems. Therefore, it is important to check the contact stress between cam and tappet. Considering the cam and tappet as linear contact of metal cylinder, the contact stress can be calculated as below:

$$
\sigma_{c}=0.564 \sqrt{\frac{F\left(\frac{\rho_{1}+\rho_{2}}{\rho_{1} \rho_{2}}\right)}{\left(\frac{1-u_{1}^{2}}{E_{1}}+\frac{1-u_{2}^{2}}{E_{2}}\right) W}}
$$

Where: $\mathrm{F}$ is the normal force between the cam and tappet, $\rho_{1}$ and $\rho_{2}$ are radius of curvature at the contact point of the cam and tappet, $E_{1}$ and $E_{2}$ are elastic modulus of the cam and tappet, $\mathbf{u}_{1}$ and $\mathbf{u}_{2}$ are the Poisson's ratios, $\mathrm{W}$ is the contact width of the cam and tappet.

\section{Multidisciplinary Design Optimization of the Distribution Cam Mechanism}

\subsection{Design variables}

Currently, the general method of the cam linear optimization is using high-order curve to fit the cam working segment. However, the cam linear cannot be express well by normal high-order curve for it is complex. It is proposed to divide the working segment of the cam into several parts, and select the distance according to different angle as the design variables in this article. Then, spline curve is applied to fit the cam working segment (Figure 6).

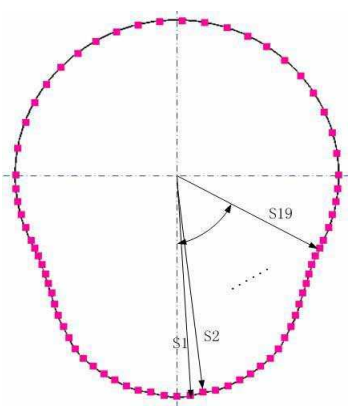

Figure 6: Design variables of the cam

\subsection{MDO of the distribution cam mechanism for multi objectives}

Because the optimization process involves coupled multiple disciplines, traditional methods for single target are no longer meet the requirement of improving the overall performance of diesel engine. The 
multi-objectives optimization model of the DCM can be founded [13]:

$$
\begin{cases}\operatorname{Max}( & F M X S), \operatorname{Min}(M A X F) \\ \text { s.t. } & M A X a \leq 667.790 m / s^{2}, \\ & M I N a \geq-11528.3 m / s^{2}, \\ & M A X F \leq 6073.837 N, \\ & S 1>S 2>\cdots>S 19\end{cases}
$$

Where: $\mathrm{S} 1, \mathrm{~S} 2, \cdots, \mathrm{S} 19$ are the design variables of the cam (see Figure 6), MAXa and MINa are the max and min acceleration of the valve, MAXF is the maximum contact force between cam and tappet, FMXS is the fullness coefficient of the valve. Thus the optimization problem changes into a two-target multidisciplinary design optimization problem.

\subsection{Solving of the MDO problem}

In order to solve the problem proposed in section 3.2, the optimization process is integrated under the environment of MDO (see Figure 7). The MDO task can be divided into three steps. Firstly, build the parametric model of the DCM in ADAMS and complete the dynamic analysis; secondly, obtain the dynamic results such as acceleration and velocity of the system, calculate the fullness coefficient and get the largest contact; at last, integrate the optimization task under the MDO environment.

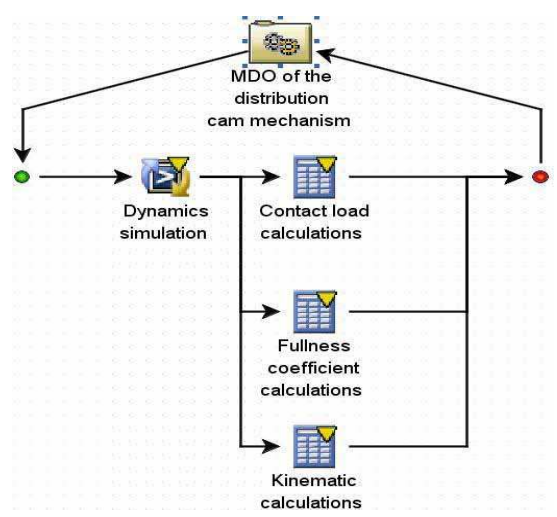

Figure 7: Process integration of the MDO of the distribution cam mechanism

According to the mathematic model of multi-objective optimization, the solving of the problem includes the following steps: (1) Searching for optimal solution. Because there are several design variables and more than one optimization objective, the process of searching for the optimal solution is difficult. Local optimal solution may be obtained for each calculation is independent. Therefore, the multi-island genetic algorithm is employed to solve the multi-objective problems in this article. (2) Selecting the optimal solution. To multi-objective optimization, participation of the decision makers is needed. The general method of selecting optimal solution is setting the weight to every objective according to requirement of the designer, and then changes the multi-objective problems to single objective problem.

\subsection{Application of approximate model}

The solution of the optimization problem discussed above is inefficiency because of the time-consuming simulation analysis and complex iteration process. However, the efficiency is important to the design of complex products. In this paper, approximate model is applied to improve the efficiency. As shown in Figure 8, the approximate model constructs mathematical function in design space to express the relationship between the design variables and the system response. In the other word, simulation analyses are replaced by approximate model basing mathematical functions for cutting down the design and analysis time. First the approximation model is optimized, and then the approximate optimum is given as the input to the exact simulation code. The approximation model is often referred to as the low fidelity model while the exact model is referred to as the high fidelity model [10].

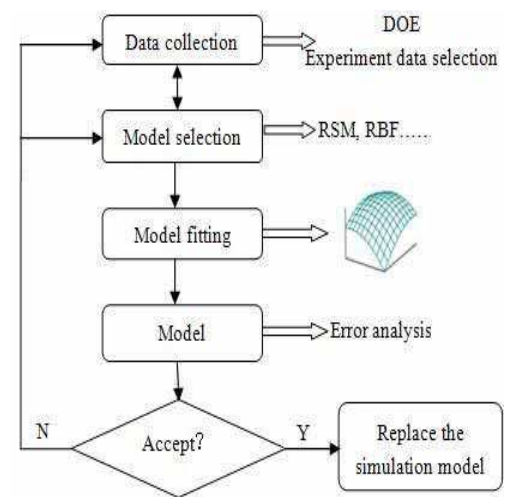

Figure 8: Design flow of MDO based approximate model

The common approximate models are response surface model (RSM), Radial basis function neural network model (RBS) and so on. RSM is based on the knowledge of statistics and mathematic and using simple mathematical expressions (commonly the lower level polynomial) take the place of actual analysis model. Here, two-level polynomial model is selected:

$$
\mathbf{F}(X)=a_{0}+\sum_{i=1}^{N} b_{i} x_{i}+\sum_{i=1}^{N} c_{i i} x_{i}^{2}+\sum_{i j(i<j)} c_{i j} x_{i} x_{j}
$$

Here $\mathrm{N}$ is the number of input various, $x_{i}$ is the $i^{t h}$ input various, $\mathrm{a}, \mathrm{b}$ and $\mathrm{c}$ are the polynomial coefficients.

\subsection{Results and discussion}

In order to contrast the results with multi-objective optimization, single objective (fullness coefficient) 
optimization is also completed using the sequential quadratic programming. The optimization iterative process is shown in Figure 9, and the contrast of optimization variables, constraints and objectives are list in Table 2 and Table 3. It can be noticed that: the valve of maximum acceleration changed from $667.79 \mathrm{~m} / \mathrm{s}^{2}$ to $549.93 \mathrm{~m} / \mathrm{s}^{2}$, minimum acceleration changed from $-11528.30 \mathrm{~m} / \mathrm{s}^{2}$ to $-5935.94 \mathrm{~m} / \mathrm{s}^{2}$ which means the movement of the system becomes more stable; the contact force reduces about 17.6 percent which reduced wear; the fullness coefficient is reduced to 0.642 and increased the intake and exhaust performance.

The optimal solution set for multi-objective (fullness coefficient and contact performance) is shown in Figure 10 and some of the results are listed in Table 2 and Table 3. Compared to single-target optimization, the optimization variables change a little, but the maximum contact force reduced $281.52 \mathrm{~N}$ and the fullness coefficient increased 0.112 , which indicates that the multi objective optimization obtains much better results.

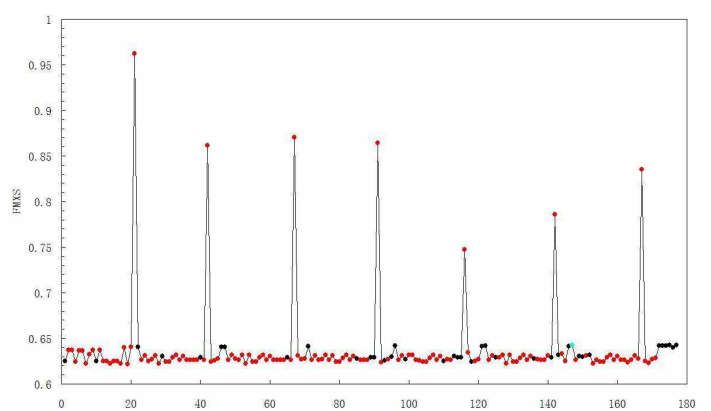

Figure 9: Optimization iterative processes for single target

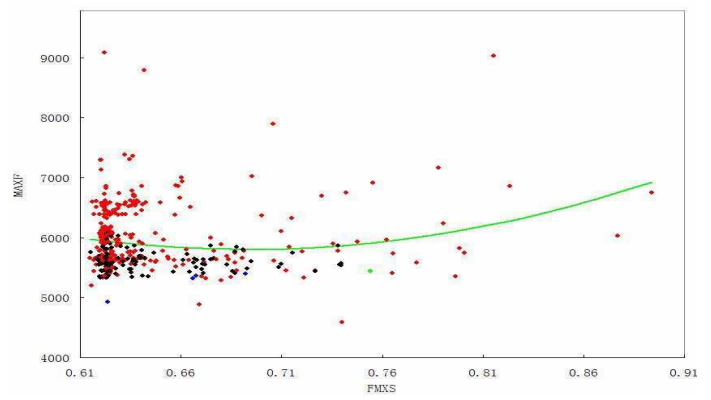

Figure 10: Optimal solution set for multi-objective

Table 2: Comparison of the optimization result of single-target and multi-target

\begin{tabular}{cccccc}
\hline Variables & $S 1$ & $S 2$ & $\cdots$ & $S 18$ & $S 19$ \\
\hline Initial value & 27.152 & 26.871 & & 19.136 & 19.059 \\
Single-target & 27.137 & 26.873 & & 19.152 & 19.071 \\
Multi-target & 27.152 & 26.871 & & 19.139 & 19.054 \\
\hline
\end{tabular}

Table 3: Comparison of the results of constraint and objective

\begin{tabular}{ccccc}
\multicolumn{5}{c}{ functions } \\
\hline Variables & MAXa & MINa & MAXF & FMXS \\
\hline Initial value & $668 m / s^{2}$ & $-11528 m / s^{2}$ & $6074 N$ & 0.625 \\
Single target & $550 m / s^{2}$ & $-5936 m / s^{2}$ & $5719 N$ & 0.6423 \\
Multi-target & $494 m / s^{2}$ & $-6506 m / s^{2}$ & $5437 N$ & 0.7543 \\
\hline
\end{tabular}

\section{Conclusions}

(1) Dynamic and kinematic analysis of the diesel engine distribution cam mechanism is completed and the intake and exhaust performance is obtained;

(2) MDO is employed in the design of diesel engine structure basing multi-objective genetic algorithms which shown good results. Cam linear parameters are selected as design variables, fullness coefficient and contact performance are set as targets, and then the optimization model is obtained under the environment of MDO for multi-objective;

(3) Compared to single target method, multi-objective optimization obtains better results, and the computational efficiency is greatly improved by application of approximate model.

\section{Acknowledgement}

This work is supported in part by National Defense Basic Scientific Research program of ChinaA3820110003

\section{References}

[1] H. Z Huang, X. L Zhang and W Yuan, Collaborative Reliability Analysis under the Environment of Multidisciplinary Design Optimization Concurrent Engineering: Research and Applications, 19, 245-254 (2011).

[2] T. L Ma and D. L Ma, Multidisciplinary DesignOptimization Methods for Aircrafts using LargeScale System Theory, Systems Engineering-Theory \& Practice, 29, 186-192 (2009).

[3] J. Roshanian, Z. Keshavarz, Effect of Variable Selection on Multidisciplinary Design Optimization: a Flight Vehicle Example Chinese Journal of Aeronautics, 20, 86-96 (2007).

[4] W Yao, X. Q Chen, W. C Luo, et al, Review of uncertainty-based multidisciplinary design optimization methods for aerospace vehicles Progress in Aerospace Sciences, 47, 450-479 (2011).

[5] M. Balesdent, et al, A survey of multidisciplinary design optimization methods in launch vehicle design, Struct Multidisc Optim, 45, 619-642 (2012).

[6] X. G Song and L Wang, Multidisciplinary optimization of a butterfly valve, ISA Transactions, 48, 370-377 (2009).

[7] Z. Y Hao and P YuOptimizing Template Design and Dynamics Simulation for the Cam Shape Line China Mechanical Engineering, 15, 913-915 (2004)

[8] L. C Long, M. Z Song, L. P Hu, et alConstruct ion and Optimization Design of a Combined Polynomial Cam Profile for Avoiding CollisionTransactions of CSICE, 20, 171-175 (2002). 
[9] Z. Li, J. B Sun and Y. G Lou, Dynamic Optimization Design of Distribution Can Mechanism in Internal Combustion Engine Based on Immune Genetic Algorithms, Journal of Vibration and Shock, 24, 68-70 (2005).

[10] M. A. Schnning and J. F. Nayfeh, P. R. Zarda, Utilization of a dependency-tracking language to reduce computational time during multidisciplinary design optimization, Advances in Engineering Software, 34, 115-122 (2003).

[11] Alexandra Ahlqvist M. Dependency-tracking objectoriented multidisciplinary design optimization (MDO) formulation on a large-scaled system, PhD Thesis. University of Central Florida, December (2001).

[12] N. H Kim, M. H Choi, S. Y Kim, et alDesign of experiment (DOE) method considering interaction effect of process parameters for optimization of copper chemical mechanical polishing (CMP) process, Microelectronic Engineering, 83, 506-512 (2006).

[13] K. H Yu, J. S Wang, X Yang, et al, Multidisciplinary Design Optimization of Cooling Turbine Blade Profiles Based on Surrogate Model, Journal of Mechanical engineering, 47, 106-112 (2011).
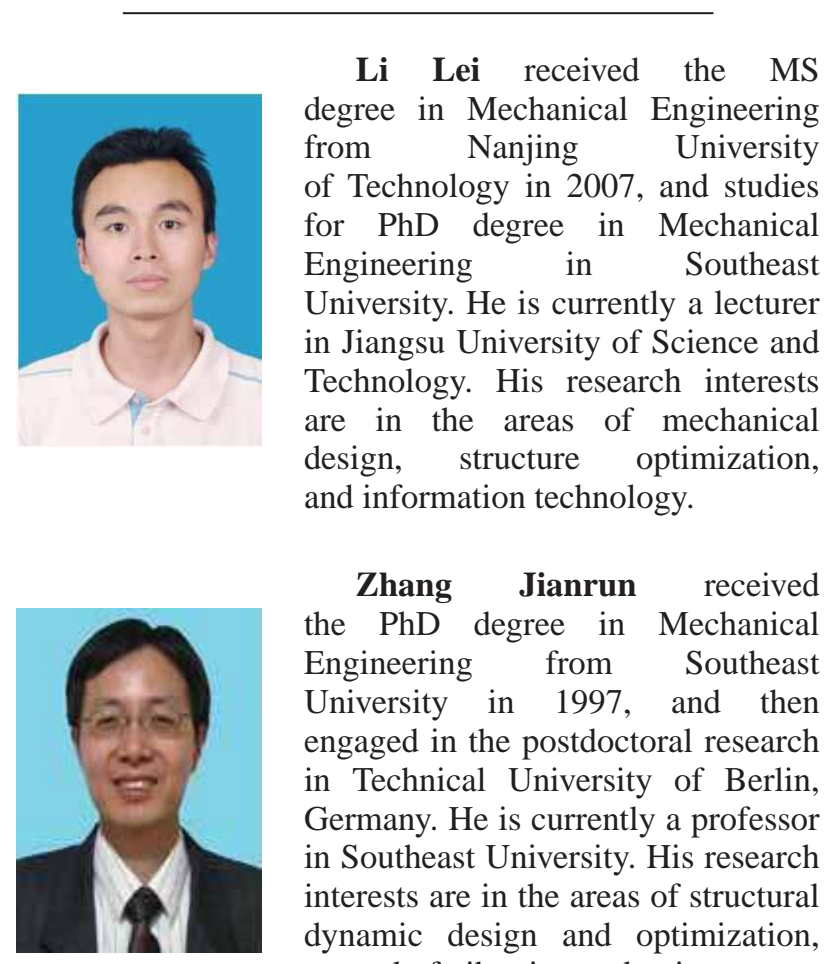

Zhang Jianrun received the $\mathrm{PhD}$ degree in Mechanical Engineering from Southeast University in 1997, and then engaged in the postdoctoral research in Technical University of Berlin, Germany. He is currently a professor in Southeast University. His research interests are in the areas of structural dynamic design and optimization, control of vibration and noise. 